Известия НАН Армении, Физика, т.57, №1, с.70-75 (2022)

УДК 548.0

DOI:10.54503/0002-3035-2022-57.1-70

\title{
ДИФРАКЦИЯ ФРАУНГОФЕРА НА ЩЕЛИ В ОДНОРОДНУЮ АНИЗОТРОПНУЮ СРЕДУ
}

\author{
О.С. ЕРИЦЯН ${ }^{1}$, А.А. ЛАЛАЯН ${ }^{2 *}$, А.Л. МАРГАРЯН ${ }^{1}$, \\ А.Г. МКРТЧЯН ${ }^{1}$, Ж.Б. ХАЧАТРЯН ${ }^{1}$ \\ ${ }^{1}$ Институт прикладных проблем физики НАН Армении, Ереван, Армения \\ ${ }^{2}$ Ереванский государственный университет, Ереван, Армения \\ *e-mail: alalayan@ysu.am \\ (Поступила в редакцию 23 сентября 2021 г.)
}

\begin{abstract}
Рассмотрена Фраунгоферова дифракция света на щели на непрозрачном экране, расположенном между вакуумом и однородной анизотропной средой в отсутствие и в присутствии поглощения при различных ориентациях оптической оси среды относительно щели. Обобщена известная формула дифракции в вакууме, включающая также случай дифракции в одноосную анизотропную среду, в отсутствие поглощения. Получена формула, позволяющая определять направления дифракционных минимумов и максимумов с учетом поглощения.
\end{abstract}

\section{1. Введение}

Хотя принципы дифракции в оптике установлены давно [1-4], рассмотрение ситуаций, когда дифрагированное излучение проникает не в вакуум, как обычно, а в материальную среду, представляет интерес ввиду того, что среда, в которой распространяется дифрагированное излучение, оставляет свой отпечаток на картину дифракции, что выступает поводом, по выражению Б.М. Болотовского «смотреть на старые явления с новой точки зрения». Так, в отличие от стандартного случая, когда по обе стороны щели - вакуум, в случае распространения излучения после щели в двулучепреломляющую среду формируется не одна, а две серии максимумов и минимумов [5]. Учет особенностей дифракции света в анизотропную среду весьма актуален в задачах создания новых микронных и наноразмерных структур с применением дифрактивных оптических и рентгеновских методов [6,7], а также для дифракционного анализа различных материалов, в том числе в современной наноскопии [8-10].

В настоящей работе рассматривается Фраунгоферова дифракция на щели в однородную среду, обладающую двойным линейным преломлением. При этом среда считается оптически одноосной. Ввиду распространения двух линейно поляризованных волн, которым соответствуют разные показатели преломления, в среде, в результате дифракции, формируются две серии максимумов и минимумов, соответствующие обыкновенной и необыкновенной волнам. 
В параграфе 2 получена формула для направлений дифракционных минимумов. Обсуждаются ситуации, когда оптическая ось среды перпендикулярна плоскости щели, когда перпендикулярна границам щели, будучи параллельной плоскости щели, а также когда параллельна границам щели.

В параграфе 3 рассмотрена задача учета поглощения при дифракции в анизотропную и изотропную среду. В конце работы приведены выводы и заключение.

\section{2. Фраунгоферова дифракция в непоглощающую анизотропную среду}

Рассмотрим простейший случай анизотропной среды, когда нет поглощения. Так как каждый пучок, соответствующий минимуму, формируется суммированием полей элементарных пучков, которые все имеют одно и тоже направление, задаваемое углом $\theta$ в фундаментальной формуле [4]

$$
d \sin \theta=m \lambda .
$$

Условие минимумов для анизотропной среды без поглощения, на основании выражения (1) может быть записано в том же виде, однако, при этом, любому направлению $\theta$ соответствует своя длина волны $\lambda(\theta)$ :

$$
d \sin \theta=m \lambda(\theta) .
$$

Обозначив компоненты тензора диэлектрической проницаемости среды за щелью через $\varepsilon_{\|}$и $\varepsilon_{\perp}$ (соответственно вдоль и поперек оптической оси), рассмотрим три простейших случая дифракции Фраунгофера в одноосную двулучепреломляющую среду.

a) Оптическая ось перпендикулярна плоскости щели (рис.1).

Для волнового вектора имеем:

$$
\frac{k_{x}^{2}}{\varepsilon_{\|}}+\frac{k_{Z}^{2}}{\varepsilon_{\perp}}=\frac{\omega^{2}}{c^{2}},
$$

где $\varepsilon_{\|}$и $\varepsilon_{\perp}-$ компоненты диэлектрической проницаемости среды

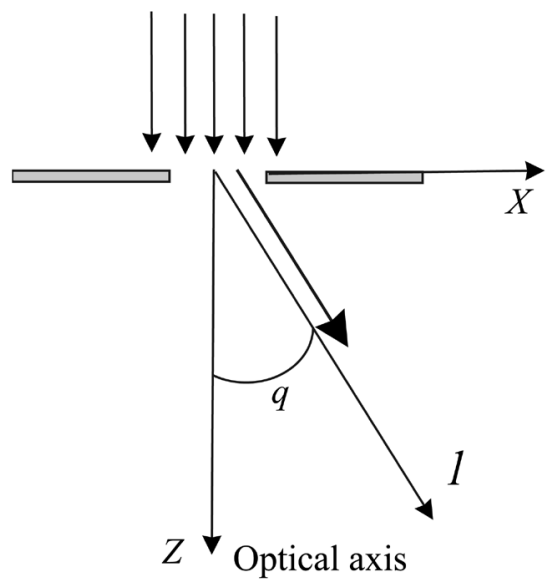

Рис.1. Дифракция Фраунгофера в одноосную двулучепреломляющую среду, в случае, когда оптическая ось перпендикулярна плоскости щели. Стрелкой 1 показано направление распространения дифрагированного пучка, жирной стрелкой - волновой вектор волны имеющий направление 1 . 
соответственно в направлениях, параллельной и перпендикулярной оптической оси. Записав (3) в виде:

$$
k^{2}\left(\frac{\sin ^{2} \theta}{\varepsilon_{\|}}+\frac{\cos ^{2} \theta_{e}}{\varepsilon_{\perp}}\right)=\frac{\omega^{2}}{c^{2}},
$$

для длины волны будем иметь

$$
\lambda_{e}(\theta)=\lambda_{o}\left\{\frac{\sin ^{2} \theta}{\varepsilon_{\|}}+\frac{\cos ^{2} \theta_{e}}{\varepsilon_{\perp}}\right\}^{1 / 2},
$$

где $\lambda_{\mathrm{o}}$ длина волны в вакууме, и фундаментальная формула (1) запишется в виде

$$
\begin{gathered}
d \sin \theta_{e}=m \lambda_{o}\left\{\frac{\sin ^{2} \theta}{\varepsilon_{\|}}+\frac{\cos ^{2} \theta_{e}}{\varepsilon_{\perp}}\right\}^{1 / 2}, \\
d \sin \theta_{o}=m \frac{\lambda_{o}}{\sqrt{\varepsilon_{\perp}}},
\end{gathered}
$$

для необыкновенной и обыкновенной волн соответственно.

В таблице 1 приведены значения $\theta$ для первых пяти дифракционных минимумов соответствующие обыкновенной и необыкновенной волнам, рассчитанных для двулучепреломляющего кристалла кварцита при $\varepsilon_{\|}=2.7502$ и $\varepsilon_{\perp}=2.2093$.

Табл.1.

\begin{tabular}{|c|c|c|}
\hline $\begin{array}{c}\text { Порядок } \\
\text { дифракции }\end{array}$ & $\begin{array}{c}\theta, \text { радиан } \\
\text { необыкновенная волна }\end{array}$ & $\begin{array}{c}\theta, \text { радиан } \\
\text { обыкновенная волна }\end{array}$ \\
\hline$m=1$ & 0.0000475726 & 0.0000336389 \\
\hline$m=2$ & 0.0000951453 & 0.0000672779 \\
\hline$m=3$ & 0.000142718 & 0.00010091690 \\
\hline$m=4$ & 0.0001902907 & 0.0001345558 \\
\hline$m=5$ & 0.0002378634 & 0.000168195 \\
\hline
\end{tabular}

Как видно из таблицы, после щели в анизотропной двулучепреломляющей среде формируются две серии максимумов и минимумов с различными углами $\theta$.

b) Оптическая ось перпендикулярна к границам щели (рис.2).

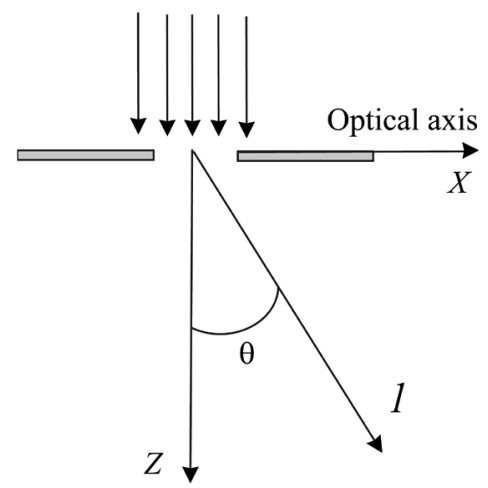

Рис.2. Дифракция Фраунгофера в одноосную двулучепреломляющую среду, в случае, когда оптическая перпендикулярна к границам щели. 
В этом случае $\varepsilon_{\|}$и $\varepsilon_{\perp}$ в выражении (6а) должны поменяться местами

$$
d \sin \theta_{e}=m \lambda_{o}\left\{\frac{\sin ^{2} \theta}{\varepsilon_{\perp}}+\frac{\cos ^{2} \theta_{e}}{\varepsilon_{\|}}\right\}^{1 / 2},
$$

а выражение (6b), соответствующее обыкновенной волне, остается неизменной.

c) Оптическая ось параллельна границам щели (рис.3).

В этом случае для необыкновенной волны мы должны подставить $\theta=\pi / 2$, и будем иметь из (6а)

$$
d \sin \theta_{e}=m \frac{\lambda_{\mathrm{o}}}{\sqrt{\varepsilon_{\|}}}
$$

а выражение (6b), соответствующее обыкновенной волне, остается неизменной.

Для изотропной среды с показателем преломления $\sqrt{\varepsilon}$ справедливо соотношение (6b) для обыкновенной волны, если заменить $\varepsilon_{\perp}$ на $\varepsilon$.

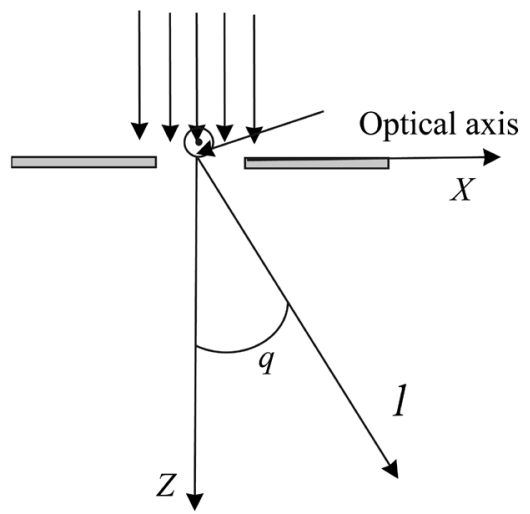

Рис.3. Дифракция Фраунгофера в одноосную двулучепреломляющую среду, в случае, когда оптическая ось параллельна границам щели.

\section{3. Учет поглощения}

При наличии поглощения элементарные пучки, идущие в одном и том же направлении из разных участков щели, имеют не только разные фазы, как в отсутствие поглощения, но и разные амплитуды, тем меньшие по величине, чем больше геометрический путь, пройденный данным элементарным пучком. Поэтому при их суммировании следует учитывать, кроме различия фаз, также различие амплитуд элементарных пучков, распространяющихся в одном и том же направлении. Примем фазу бесконечно узкого пучка, идущего с середины щели, равной нулю (см. рис.4). Фаза бесконечно узкого пучка, идущего из точки, смещенной на х от середины щели, будет равна $k_{\theta} x \sin \theta$ где $k_{\theta}-$ модуль волнового вектора, составляющего угол $\theta$ с нормалью к плоскости щели.

Считая фазу поля в плоскости щели равной нулю, для произвольного бесконечно узкого пучка, идущего от полоски шириной $d x$, будем иметь

$$
d E=\text { const } e^{i k(\theta) x \sin \theta} d x .
$$

Обобщая подход, примененный к случаю дифракции в вакуум [4], для полного поля получим выражение пропорциональное интегралу 


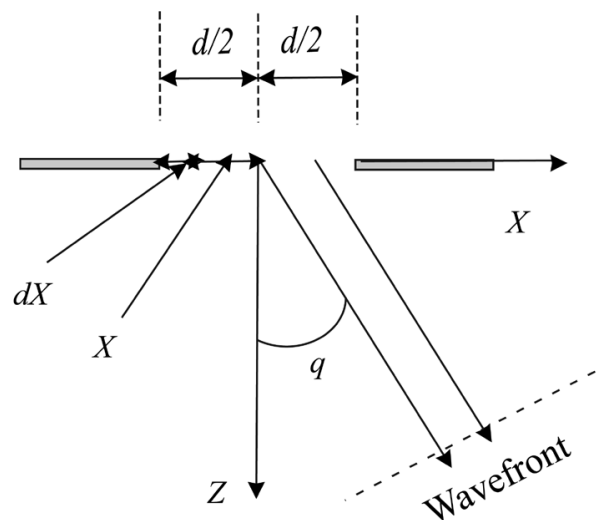

Рис.4. Дифракция Фраунгофера с учетом поглощения.

$$
I(\theta)=\int_{x=-d / 2}^{x=+d / 2} \cos \left[k^{\prime}(\theta) x \sin (\theta)\right] e^{k^{\prime \prime}(\theta) x \sin (\theta)} d x,
$$

где $k^{\prime}(\theta)$ и $k^{\prime \prime}(\theta)$ действительная и мнимая части волнового вектора волны, распространяющейся под углом $\theta$ к направлению нормали к плоскости щели. Заметим, что с ростом $x$ от $-d / 2$ до величины $+d / 2$ значение $e^{k^{\prime \prime}(\theta) x \sin \theta}$ растет в соответствии с тем, что переход $x$ от $-d / 2$ до $+d / 2$ соответствует переходу от крайне левого луча до крайне правого, тем самым длина пути луча в поглощающей среде от плоскости щели до формирования фронта уменьшается. Интегрируя, из (10) получаем для суммарного поля

$$
E=\text { const }\left.\frac{e^{b x}(b \cos a x+\sin a x)}{a^{2}+b^{2}}\right|_{x=-d / 2} ^{x=+d / 2},
$$

где

$$
a=k^{\prime}(\theta) \sin \theta, \quad b=k^{\prime \prime}(\theta) \sin \theta .
$$

Величины $k^{\prime}(\theta)$ и $k^{\prime \prime}(\theta)$ (действительная и мнимая части волнового вектора), определяются из уравнения (4) поверхности волновых векторов той среды, куда проникает дифрагированное излучение, $\theta$ - угол между волновым вектором и оптической осью среды, куда проникает дифрагированное излучение.

Как и в случае, рассмотренном в параграфе 2, следует сделать замену $\cos \theta \leftrightarrow \sin \theta$ или $\varepsilon_{\|} \leftrightarrow \varepsilon_{\perp}$, если оптическая ось перпендикулярна к границам щели. Если оптическая ось параллельна границам щели, то следует подставить $k_{e}^{2}=\frac{\omega^{2}}{c^{2}} \varepsilon_{\|}$, тогда закономерность углового распределения интенсивности идентична закономерности распределения для обыкновенной волны, если заменить $\varepsilon_{\|}$на $\varepsilon_{\perp}$.

\section{4. Заключение}

При дифракции света на щели в материальной среде картина распределения дифракционных минимумов и максимумов зависит от оптических характеристик этой среды. В работе обобщена известная формула дифракции Фраунгофера в вакууме на случай материальной среды с включением дифракции в одноосную анизотропную среду в отсутствие и присутствии поглощения. Показана 
зависимость распределения интенсивности дифрагированной волны от поляризации падающего излучения.

Авторы выражают благодарность В. Кочаряну за полезные обсуждения.

\title{
ЛИТЕРАТУРА
}

1. M. Born, E. Wolf. Principles of Optics, 4th ed., New York: Pergamon Press, 1968.

2. J.M. Cowley. Diffraction physics, Amsterdam, North-Holland, 1975.

3. Р.Б. Ваганов, Б.З. Каценелбаум. Основы теории дифракции, Москва: Наука, 1982.

4. Д.В. Сивухин. Общий курс физики, Оптика, том IV, Москва: Наука, 1980.

5. A.R. Mkrtchyan, A.A. Lalayan, H.S. Yeritsyan, A.L. Margaryan, Sh.K. Yeritsyan, Zh.B. Khachatryan. J. Contemp. Phys., 55, 314 (2020).

6. Y.E. Geints, O.V. Minin, I.V. Minin. Annalen der Physik, 531, 1900033( 2019).

7. L. Yang, et al. Nanomaterials, 9, 1789 (2019).

8. D. Xu, K.P. Chen, K. Ohlinger, et al. Nanotechnology, 22, 035303 (2011).

9. R.D. Johnson, P.G. Radaelli. Annual Review of Material Research, 44, 269 (2014).

10. S. Mourdikoudis, R.M. Pallares, N.T.K. Thanh. Nanoscale, 10, 12871 (2018).

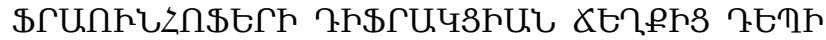

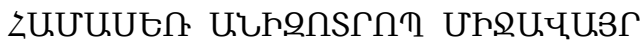

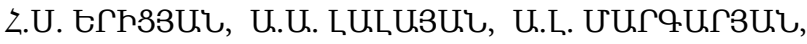

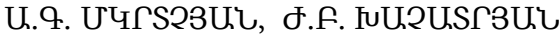

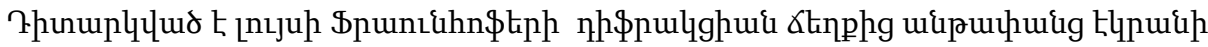

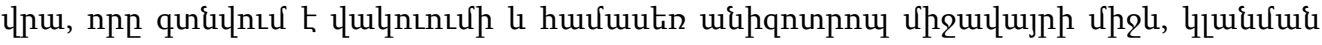

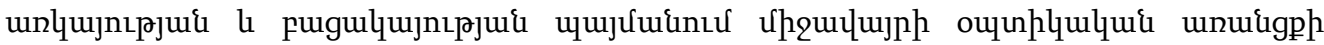

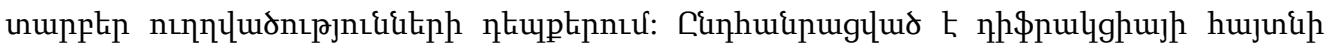

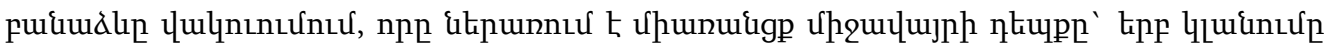

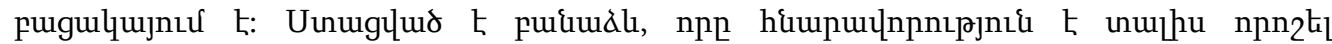

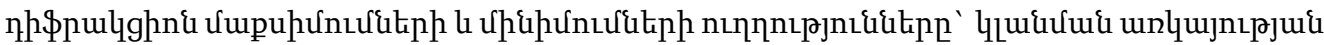
ntuppnux:
\end{abstract}

\section{FRAUNHOFER DIFFRACTION BY SLIT INTO A HOMOGENEOUS ANISOTROPIC MEDIUM}

\author{
H.S. ERITSYAN, A.A. LALAYAN, A.L. MARGARYAN, \\ A.G. MKRTCHYAN, Zh.B. KHACHATRYAN
}

Fraunhofer diffraction of light by a slit on an opaque screen located between a vacuum and a homogeneous anisotropic medium in the absence and in the presence of absorption at different orientations of the optical axis of the medium relative to the slit is considered. The well-known formula for diffraction in vacuum is generalized, which also includes the case of diffraction into a uniaxial anisotropic medium in the absence of absorption. A formula has been obtained that makes it possible to determine the directions of the diffraction minima and maxima taking into account the absorption. 